\title{
Componentização e padrões Web Services em Dispositivos Móveis utilizando LBS ${ }^{*}$
}

\author{
Alessio Miranda Júnior ${ }^{1}$, Alex Damiany Assis ${ }^{1}$, Mauro Nacif Rocha1 \\ ${ }^{1}$ Universidade Federal de Viçosa \\ alessio@juninho.com.br, adassis@dpi.ufv.br, mnacif@ufv.br
}

\begin{abstract}
Resumo. A crescente demanda por sistemas que utilizam LBS (LocationBased Services) em dispositivos móveis é algo em evidência na atualidade. $O$ objetivo deste projeto consistiu em desenvolver uma solução para facilitar a implementação de LBS e que atenda aos requisitos de portabilidade $e$ independência da linguagem de programação utilizada. Como resultado, utilizando a middleware MoCA, foi desenvolvido uma solução Web Services, criando sobre esta middleware uma nova camada de abstração, onde estão disponibilizados serviços com interface voltada para o fornecimento de LBS usando o protocolo SOAP, troca de mensagens em XML.
\end{abstract}

\section{Introdução}

O conceito de $W$-LAN trouxe benefícios e enormes possibilidades de utilização como acesso a todo tipo de informação em qualquer lugar com independência de fios, cabos e localidade. A democratização e utilização de dispositivos móveis portáteis, como notebook e pocketpc, incentivou o desenvolvimento de diversas aplicações para o público portátil e esses dispositivos que foram agregando novas tecnologias como rede $W-L A N$ tem se tornado cada vez mais comum.

Inicialmente limitado, os sistemas de informação para usuários móveis sem fio se enquadravam num modelo muito simples, sendo basicamente textual e específico para determinados segmentos. Avanços tecnológicos tornaram estes dispositivos cada vez menores e com maior capacidade de processamento; é notável a necessidade de sistemas mais robustos, utilizando recursos multimídia e web, sem esquecer de questões criticas como consumo de energia, interferência e segurança.

Dentro deste contexto o desenvolvimento de sistemas com LBS (Location-Based Services) [SCHILLER, 2004], usando tecnologia móvel tem se tornado uma área bastante interessante para estudos práticos. A demanda por estes novos sistemas tem sido ampliada, e com a democratização da tecnologia W-LAN, que hoje já é implantada nos mais diferentes lugares, domicílios, empresas e locais de domínio público como aeroportos e shopping centers. Isso amplia as possibilidades de aplicações de acesso à informação, colaboração e comunicação.

\footnotetext{
* Este trabalho recebeu suporte da FAPEMIG, na modalidade "auxílio à pesquisa", Processo $\mathrm{N}^{\circ}$ EDT249/05
} 
Buscamos neste trabalho criar formas de facilitar o desenvolvimento de sistemas baseados em localização, de forma que seja facilmente utilizado por qualquer tecnologia, linguagem de programação ou plataforma, assim adotamos a estratégia de desenvolvimento de Web Services [STAL, 2002], onde podemos prover serviços com semântica específica, os quais poderão ser utilizados por uma grande variedade de aplicações. Na base destes serviços foi utilizada uma middleware [AMJAD, 2004], o MoCA [SACRAMENTO, 2004].

\section{Conceitos}

\subsection{LBS}

LBS (Serviços Baseados em Localização) é um conceito que denota aplicações que interagem com a localização geográfica, que vem crescendo e foca prover informações geográficas e espaciais via dispositivos móveis e similares. O clássico serviço por traz de um LBS é a inferência de localização, sendo que a tecnologia mais conhecida é o GPS (Sistema de Posicionamento Global) [SCHILLER, 2004]. Outra tecnologia é a triangulação de sinal de antenas, que é utilizado nos serviços de telefonia celular e também podem servir para definir a localização do usuário.

A tecnologia abordada neste trabalho será a análise de sinais (FINGERPRINTING), que consiste na inferência de localização baseada em sinais de rádio freqüência de pontos de acesso W-LAN. Fazendo o mapeamento de sinais em diferentes locais e seu armazenamento, pode-se comparar a intensidade do sinal atual com os padrões armazenados, retornando a localização aproximada do dispositivo móvel.

\subsection{MoCA}

A middleware MoCA, visa fornecer um ambiente para desenvolvimento de aplicações colaborativas móveis com serviços para coleta, agregação e acesso a diferentes informações de contexto, que podem ser disponibilizados ou utilizados por outras aplicações.

A arquitetura consiste de APIs e de um framework para facilitar a integração das aplicações. Várias aplicações de colaboração poderiam se beneficiar dos serviços da arquitetura. A arquitetura MoCA foi projetada para ambientes de redes semi-infraestruturadas. $\mathrm{O}$ atual protótipo da arquitetura foi implementado para uso em uma rede baseada em TCP/IP 802.11 mas, em princípio, nada impede que ela seja estendida para redes celulares, como GPRS.

Na arquitetura, cada aplicação de colaboração é composta de três partes: Servidor, Proxy e Cliente da Aplicação. As duas primeiras executam na rede fixa e a última no dispositivo móvel.

A Figura 1 mostra uma aplicação composta de um servidor, um ou mais proxies que atendem um conjunto de clientes em dispositivos móveis e que interagem com serviços MoCA através de eventos assíncronos usando o paradigma publish/ subscribe. 


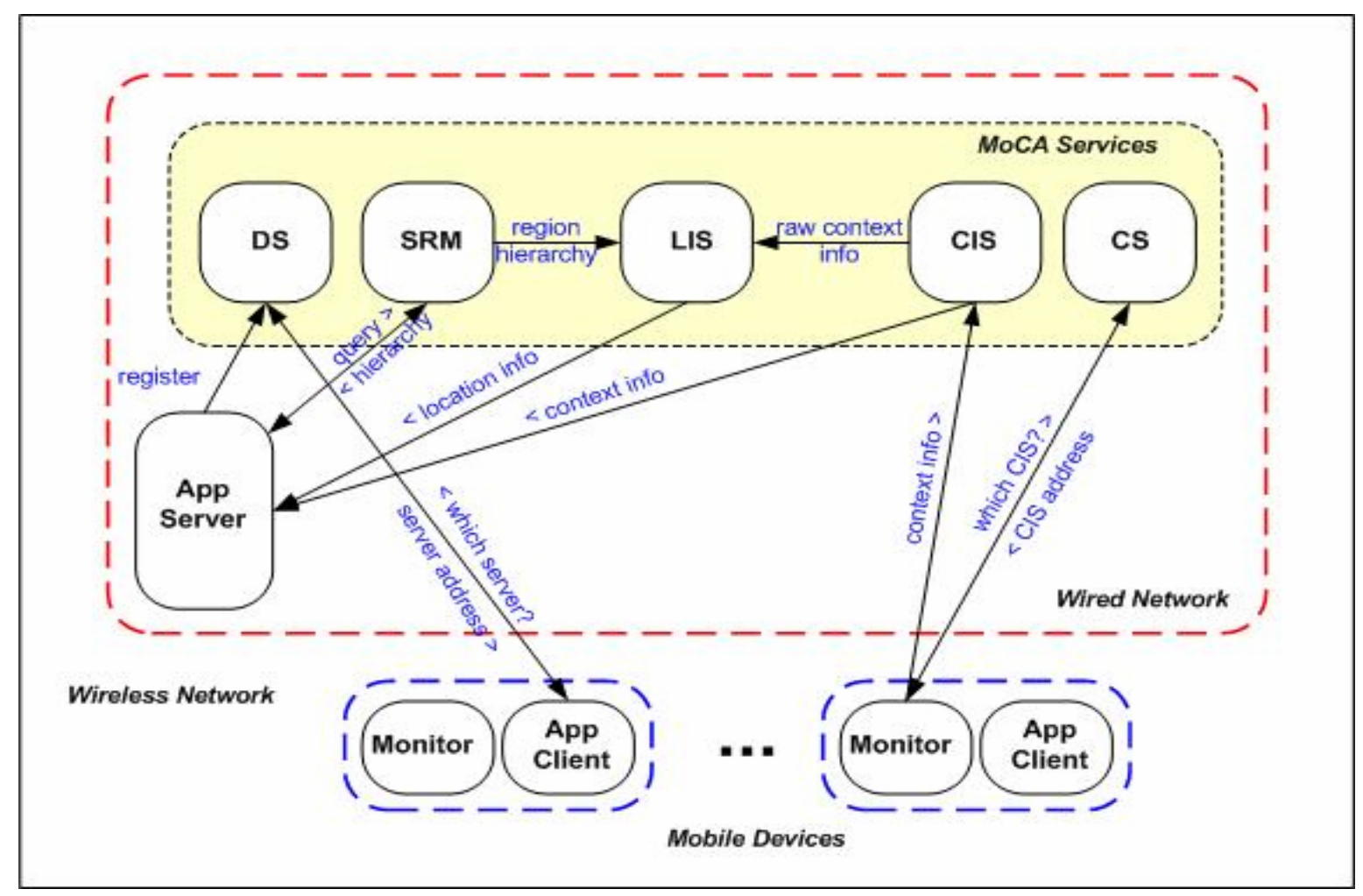

Figura 1 Arquitetura da Middleware MoCA

\subsection{SOA (Arquitetura Orientada a Serviço).}

Service-Oriented Architecture (SOA) [ERL, 2005], - ou em português, Arquitetura Orientada a Serviços - é um termo que descreve duas coisas muito diferentes. As duas primeiras palavras expressam uma metodologia para desenvolvimento de software. A terceira palavra é um panorama de todos os ativos de software de uma empresa, assim como uma planta arquitetônica é uma representação de todas as peças que, juntas, formam uma construção. Portanto, "service-oriented architecture” é uma estratégia que proclama a criação de todos os ativos de software de uma empresa via metodologia de programação orientada a serviços.

Com SOA as aplicações podem possuir grande flexibilidade para mudar ou criar processos de negócios com rapidez. Conquistar tal nível de flexibilidade costumava ser desperdício de tempo, dinheiro e mão de obra. Dependia da construção de seus próprios sistemas de integração personalizados. No entanto, o atual desenvolvimento em padrões da Internet facilita a comunicação entre aplicativos de software.

SOA se baseia na capacidade de identificar serviços e suas características. Conseqüentemente, esta arquitetura depende de um diretório que descreva quais os serviços disponíveis dentro de um domínio.

\section{Projeto}

Após estudo sobre as soluções já disponíveis, optamos pela utilização da API MoCA, pois este já provê um bom serviço de inferência de localização indoor e é capaz de atender as necessidades das Aplicações Móveis Colaborativas propostas. A API MoCA foi desenvolvida em Java tendo a característica de ser portável, porém apresenta 
problemas quando são levantados quesitos como flexibilidade de utilização por outras linguagens. Buscando solucionar estes problemas criando uma nova camada de abstração sobre a middleware, utilizamos a tecnologias Web Services, objetivando atender todo tipo de sistema.

Web Services são identificados por uma URI (Uniform Resource Identifier) [RFC 2396IETF, 1998], e são descritos e definidos usando XML [BRAY, 2004]. Um dos motivos que tornam Web Services atrativos é o fato deste modelo ser baseado em tecnologias standards, em particular XML e HTTP. Web Services são usados para disponibilizar serviços interativos na Web, podendo ser acessados por outras aplicações. SOAP (Simple Object Access Protocol) [MITRA, 2003], está se tornando padrão para a troca de mensagens entre aplicações e Web Services, já que é uma tecnologia construída com base em XML e HTTP.

SOAP é um protocolo projetado para invocar aplicações remotas através de RPC (Remote Procedure Calls - Chamadas Remotas de Procedimento) ou trocas de mensagens, em um ambiente independente de plataforma e linguagem de programação. SOAP é, portanto, um padrão normalmente aceito para utilizar-se com Web Services. Desta forma, pretende-se garantir a interoperabilidade e intercomunicação entre diferentes sistemas, através da utilização de uma linguagem (XML) e mecanismo de transporte (HTTP) padrões.

\subsection{Arquitetura}

A arquitetura é composta por um servidor onde estão em execução os serviços do MoCA: LIS (Location Information Service), CIS (Context Information Service) e SRM (Symbolic Region Manager) e o Web Service desenvolvido que disponibiliza esses serviços para acesso via Web ou por uma aplicação que utilize o padrão SOAP ou troca de mensagens XML.

O monitor é instalado no dispositivo móvel que se quer monitorar, através da rede Wireless o monitor envia os dados do dispositivo para o CIS, e este resolve com o serviço LIS a localização do dispositivo móvel. 




Figura 2 - Arquitetura desenvolvida no projeto WSDpiMoca

\subsection{Mapeamento}

O mapeamento do departamento de informática (DPI), ambiente de testes, foi feito utilizando a ferramenta Mapper criada para ajudar os usuários do LIS a fazer os mapas para inferência de localização. Esses mapas consistem do sinal RF coletado de todos Acess Point 802.11 acessíveis.

\subsection{Serviços Implementados}

Os serviços disponibilizados pelo Web Services têm o propósito de permitir que aplicações clientes usem a API MoCA, para solicitar serviços relacionados com os dispositivo móveis, que possuem tecnologia $\mathrm{Wi}-\mathrm{Fi}$, dentro da rede Wireless do Departamento de Informática (DPI) da Universidade Federal de Viçosa. Os serviços a serem implementados são divididos em dois tipo de serviços, os síncronos e assíncronos, neste trabalho nos focamos em implementar principalmente serviços síncronos. Segue a descrição dos serviços implementados.

\subsubsection{MobileLocationbyName(N)}

Esse serviço retorna a região da localização do dispositivo móvel. O parâmetro N é o nome do dispositivo móvel.

\subsubsection{MobileLocationbyMACAddress(A)}

Esse serviço retorna a região da localização do dispositivo móvel. O parâmetro A é o endereço MAC da placa de rede Wi-Fi do dispositivo móvel. (Figura 3) 


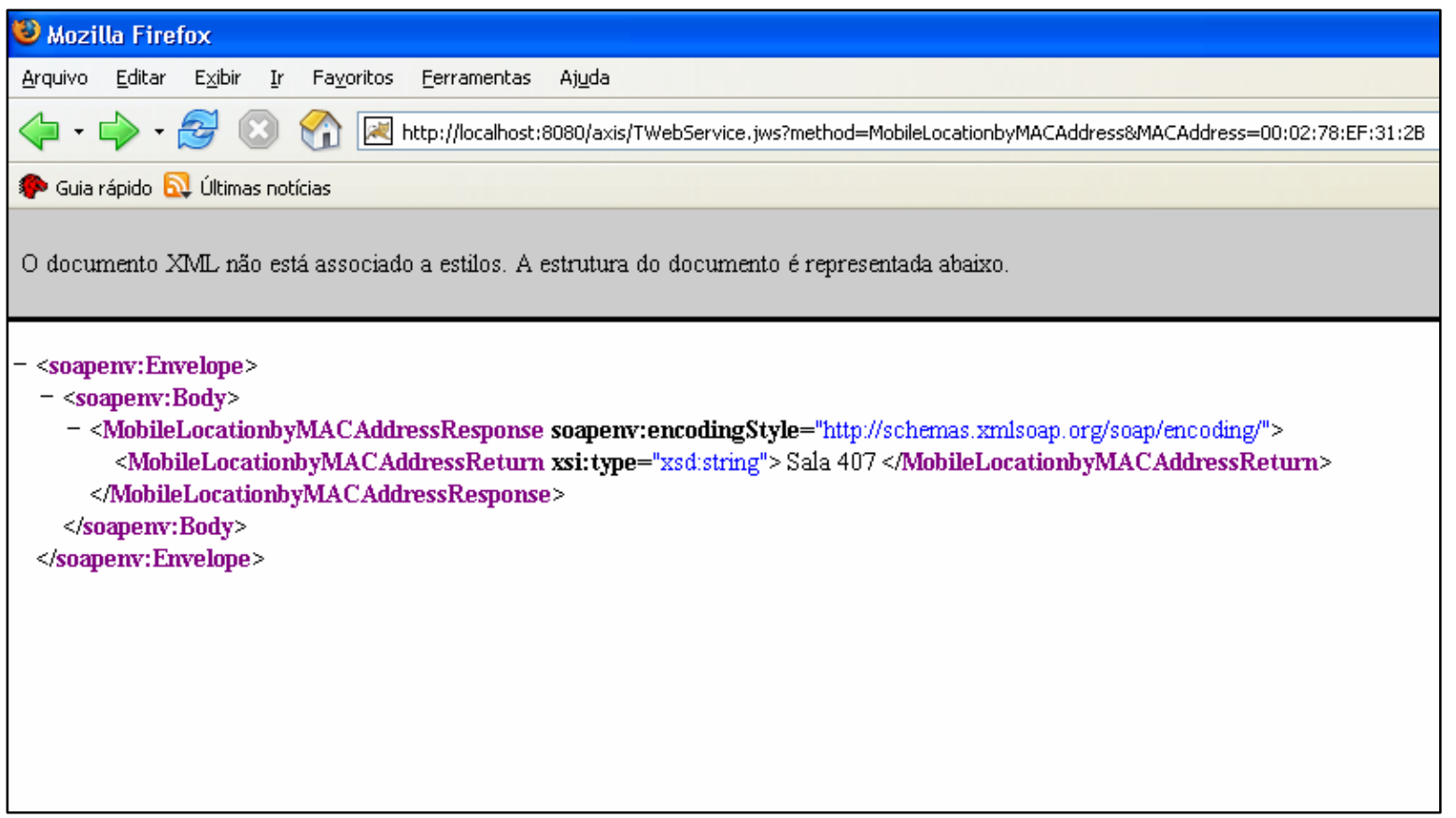

Figura 3: Resultado da invocação do serviço "MobileLocationbyMACAddress" via browser. $O$ resultado é um XML que com a localização do dispositivo.

\subsubsection{StatusMobileDevicebyName(N)}

Esse serviço retorna informações do dispositivo móvel como: uso da CPU, memória livre, energia, endereço IP, endereço MAC e endereço MAC do ponto de acesso. O parâmetro $\mathrm{N}$ é o nome do dispositivo móvel.

\subsubsection{StatusMobileDevicebyMACAddress(A)}

Esse serviço retorna informações do dispositivo móvel como: uso da CPU, memória livre, energia, endereço IP, endereço MAC e endereço MAC do ponto de acesso. O parâmetro A é o endereço MAC da placa de rede Wi-Fi do dispositivo móvel.

\subsubsection{ListAllRegion()}

Esse serviço retorna uma lista com todas as regiões mapeadas.

\subsubsection{AllHierarquy()}

Esse serviço retorna uma lista com a hierarquia das regiões.

\subsubsection{ListMobileDevice()}

Esse serviço retorna uma lista dos endereços MAC dos dispositivos móveis monitorados. 


\section{Conclusão}

O conjunto de tecnologias e arquitetura utilizada na implementação do projeto, chamado de WSDpiMoCA (Web Services DPI Moca), atingiu seu objetivo final. O WSDpiMoCA provê serviços relevantes, fácil utilização e seu uso é independente da linguagem a ser utilizada na implementação do cliente que utiliza os serviços, isto devido a utilização do SOAP como padrão de comunicação entre a aplicação cliente e o servidor.

O WSDpiMoca ainda é um modelo simples, mas com grande perspectiva de expansão. A ampliação do sistema com o desenvolvimento de novas funcionalidades e o suporte a serviços de mensagens assíncronas é uma das propostas para trabalhos futuros, tendo como outras propostas o estudo da solução para sistemas WI-Max e análise mais precisa sobre a confiabilidade dos resultados repornados pela middleware MoCA.

\section{Referências}

MITRA, NILO (2003), "SOAP Version 1.2 Primer”, http://www.w3.org/TR/soap12part0/, publicado como recomendação no W3C. Acessado em 10/01/2006.

BRAY, Tim Et al (2004) "Extensible Markup Language (XML) 1.0 (Third Edition)", http://www.w3.org/TR/2004/REC-xml-20040204/, publicado como recomendação no W3C. Acessado em 19/01/2006.

SCHILLER, JOCHEN and VOISARD, AGNÉS (2004), "Location Based Services", Cambridge University Press.

SACRAMENTO, V., ENDLER, M., RUBINSZTEJN, H. K., LIMA, L. S., GONÇAVES, K., NASCIMENTO, F. N., and BUENO, G. A. (2004), "Moca: A middleware for developing collaborative applications for mobile users.", ACM/IFIP/USENIX International Middleware Conference, Toronto, October, 2004.

MoCA (Mobile Collaboration Architecture) - http://www.lac.inf.puc-rio.br/moca, Acessado em 22/08/2006

BOOTH, DAVID (2003), "WSDL Version 2.0 Primer", http://www.w3.org/TR/wsdl20-primer/, publicado no W3C. Acessado em 26/04/2006.

ERL, THOMAS (2005), "Service-Oriented Architecture (SOA): Concepts, Technology, and Design", Prentice Hall.

AMJAD, UMAR, (2004), "Mobile Computing and Wireless Communications Applications, Networks, Platforms, Architectures, and Security, NGE Solutions".

STAL, MICHAEL (2002) "Web Services: Beyond Component-based Computing”,

PASHTAN, ARIEL, (2005), "Mobile Web Services”, Cambridge University Press.

BRUNNER, ROBERT J.; et al (2002), “Java Web Services Unleashed” - SAMS.

Communications of The ACM, Vol. 45, No 10, p. 71 - 76, Out. 2002.

RFC 2396IETF (Internet Engineering Task Force). Uniform Resource Identifiers (URI): Generic Syntax, ed. T. Berners-Lee, R. Fielding, and L. Masinter. 1998. 